\title{
PERSONAL RESOURCES, DEPRESSIVE SYMPTOMS AND SELF-RATED HEALTH IN HIGH-RISK PROFESSIONS
}

\author{
Jana Nezkusilova ${ }^{1}$, Martina Chylova $^{2}$, Livia Pestova $^{2}$, \& Monika Seilerova $^{3}$ \\ ${ }^{1}$ Department of Psychology, Faculty of Arts, P. J. Safarik University, Kosice (Slovakia) \\ ${ }^{2}$ First Department of Psychiatry, Faculty of Medicine, P. J. Safarik University, Kosice (Slovakia) \\ ${ }^{3}$ Department of Labour Law and Social Security Law, Faculty of Law, P. J. Safarik University, \\ Kosice (Slovakia)
}

\begin{abstract}
The importance of personal resources in the context of high demanding work conditions is often being reported. However, when and what type of personal resources are the beneficial ones is not fully understood. The aim of this study is to apply network analysis and explore closeness of relationships between personal resources, depressive symptoms and self-rated health in individuals working in high-risk professions. The study sample comprised 276 police officers, prison guards, customs officers and physicians $(72.1 \%$ men, Mage $=36.58$, SDage $=8.99)$. Observed variables included personal resources (OSI-R), depressive symptoms (SDS) and self-rated health (item from SF-36). The network analysis (EBICglasso) was performed in two groups: workers in emergency services $(\mathrm{N}=133)$, and individuals, who do not work in emergency services $(\mathrm{N}=143)$. Network analysis revealed that individuals who do not work in emergency services reported depressive symptoms in negative relationship to rational coping, social support, recreation and self-care. In this group, the decrease of self-rated health is related to decline of self-care, social support and recreation. Conversely, emergency workers reported their self-rated health depending solely on the level of recreation they perform. In this group, depressive symptoms could be reduced by recreation, rational coping and social support, but are not related to self-care. In the emergency workers, the self-care and self-rated health were the nodes of low strength. Therefore, intervention programs should target individuals in high-risk professions by stimulating appropriate personal resources to maintain their health. Importantly, self-care strategies might lose their protective role in emergency workers.
\end{abstract}

Keywords: Personal resources, depressive symptoms, self-rated health, emergency services, network analysis.

\section{Introduction}

Occupational stress has been recognized as a risk factor in the development of depressive symptoms (Madsen et al., 2017) and deterioration of well-being and health. In high-risk professions, stress, anxieties and depression are among common reasons leading to work absence and formal disability (Baumann et al., 2010). Night shifts, changing working hours and emergency service are related to sleep disorders, depression and health deterioration (Bara, Arber, 2009). Additionally, selected professionals police officers, prison guards, customs officers, and physicians are in direct contact with every day threatening situations. Yet, studies suggest that personal resources may protect from the development of depression (Wu et al., 2011) or health deterioration.

\section{Design and objective}

The aim of this cross-sectional study was to identify central variables that can be targeted by intervention programs to improve well-being and health in selected professions. The network analysis was utilized to compute the importance of specific variables (nodes). It is a useful tool for understanding the relationships between multiple variables - personal resources, self-rated health, and depressive symptoms in high-risk professions.

\section{Research methods}

\subsection{Participants}

The sample comprised 276 professionals from the high-risk professions. These individuals were analyzed independently based on whether they work in an emergency or not. Further description of both groups is in Table 1. The groups did not differ in any studied variable, except for gender proportion. 
Table 1. Demographic characteristics of the sample.

\begin{tabular}{|c|c|c|c|c|c|c|}
\hline \multirow{2}{*}{ Characteristic } & \multicolumn{2}{|c|}{ Non-emergency sample } & \multicolumn{2}{|c|}{ Emergency sample } & & \\
\hline & $\mathbf{N}$ & $\%$ & $\mathbf{N}$ & $\%$ & & \\
\hline \multicolumn{7}{|l|}{ Profession } \\
\hline Police officers & 46 & 32.2 & 23 & 17.3 & & \\
\hline Prison guards & 58 & 40.6 & 19 & 14.3 & & \\
\hline Customs officers & 32 & 22.4 & 33 & 24.8 & & \\
\hline Physicians & 7 & 4.9 & 58 & 43.6 & & \\
\hline \multicolumn{7}{|l|}{ Gender } \\
\hline Male & 106 & 74.1 & 93 & 69.9 & & \\
\hline \multirow{2}{*}{ Female } & 37 & 25.9 & 40 & 30.1 & & \\
\hline & $\mathbf{M}$ & SD & $\mathbf{M}$ & SD & $\mathbf{t}$ & p \\
\hline Age & 37.16 & 9.15 & 35.94 & 8.81 & 1.12 & 0.26 \\
\hline Depressive symptoms & 34.99 & 7.50 & 35.68 & 9.14 & -0.67 & 0.51 \\
\hline Self-rated health & 2.25 & 0.83 & 2.20 & 0.84 & 0.56 & 0.58 \\
\hline \multicolumn{7}{|l|}{ Personal resources } \\
\hline Recreation (RE) & 31.62 & 6.38 & 31.45 & 7.13 & 0.20 & 0,84 \\
\hline Self-care (SC) & 24.95 & 6.26 & 25.39 & 6.09 & -0.59 & 0.56 \\
\hline Social support (SS) & 40.41 & 9.42 & 41.50 & 7.85 & -1.04 & 0.30 \\
\hline Rat./Cognitive coping (RC) & 35.15 & 6.82 & 36.12 & 6.28 & -1.21 & 0.23 \\
\hline
\end{tabular}

Note. $\mathrm{M}=$ mean; $\mathrm{SD}=$ standard deviation; $\mathrm{t}=$ independent $\mathrm{t}$-test

\subsection{Measures}

Respondents fulfilled sociodemographic variables (age, gender, nightshifts, type of work), self-rated their health by a single scale and rated their depressive symptoms (20 items) in the Zung Self-Rating Depression Scale (SDS). In addition, the Occupational Stress Inventory - Revised (OSI-R, Osipow, 1998) was used. The OSI-R questionnaire measures three areas of stress in working individuals: 1. Occupational stress, 2. Psychological strain and 3. Coping resources (Personal Resources Questionnaire): Recreation, Self-Care, Social Support, and Rational/Cognitive Coping. In this study, only PRQ form OSI-R was used.

\subsection{Data analysis: network analysis}

Psychology network analysis is a form of exploratory analysis. Between each node (variable) are edges - representing the partial correlation between the two variables controlled for all other relationships in the network. The network analysis was constructed separately for individuals that do work in emergency services and those who do not offer emergency services. Both groups comprised high-risk professions - physicians, police officers, customs officers, and prison guards. Total scale scores (Zung) and total subscale scores (Self-rated health and Personal resources from OSI-R: social support, recreation, self-care, rational coping) were used for better interpretability instead of using individual item scores. The scale scores represent "nodes" in the network. The network of complex interactions between personal resources, depressive symptoms, and self-rated health was estimated using the estimation technique: graphical LASSO (Glasso). The color of the edges signifies the positive (blue color) or negative relationship (red color) between the variables and the thickness of edges represents the strength of each relationship. The used algorithm places nodes with a stronger relationship closer to the center of the graph. In the network analysis, several indices are computed to estimate the importance of each node. In this analysis, the Strength was of our main interest. The strength indicates the magnitude of the direct relationship with other nodes in the network. The estimation of the stability of the centrality indicators and the stability of edge weights - the accuracy of networks, were also computed.

\section{Results}

The stability of the centrality measures (the indicators of the importance of the nodes) revealed that the indicator of strength is stable. The analysis of the stability of edge weights performed by using 1000 bootstrap samples suggests that the tie strengths were reliably estimated. Supplementary material of difference tests between nodes centrality or edge weights may be sent upon the request.

Inspection of the network for the non-emergency and emergency employees (Figure 1, Figure 2) aiming at the strength, as a centrality measure of our main interest, reveals that the most central nodes are in both networks depressive symptoms, recreation and rational/cognitive strategies of coping. The estimated network and centrality indicators are presented in Figures 1 and 2. Self-reported (low) health was not central in the networks. Social support was not central for non-emergency employees. However, Social support was important, although with less centrality, for the group working in an emergency. On the other hand, self-care activities were of very low centrality in the group of emergency workers but were central in non-emergency workers. 
Figure 1. Estimated network of the a) non-emergency and b) emergency working group in high-risk professions.
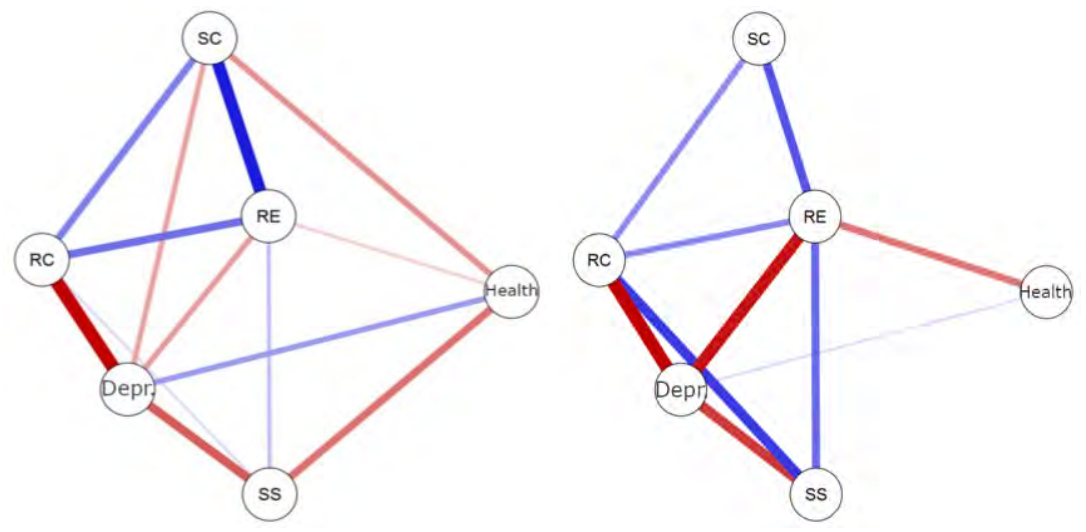

Figure 2. The Centrality Plot for emergency (red) and non-emergency (blue) group of professionals.

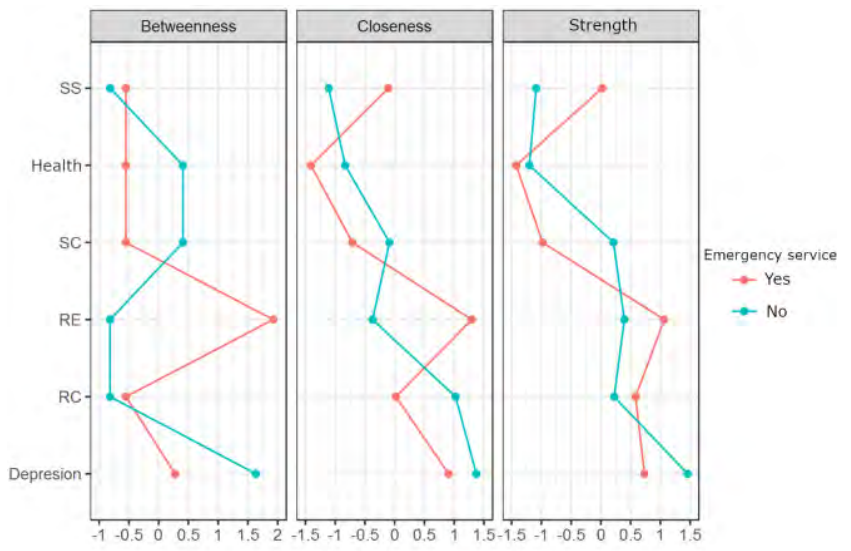

Note. Only Strength was stable indicator and was used as a centrality measure in the study.

\section{Conclusion and practical implications}

The study explored importance (centrality) of particular nodes, in this case, depressive symptoms, decrease of self-reported health as well as areas of personal resources. It can be concluded that depression, recreation and rational/cognitive strategies of coping are among the central variables that should be targeted by intervention programs supporting the health and well-being of high-risk professions as police officers, prison guards, customs officers or physicians. Additionally, intervention program makers may select participants who will benefit more from activating social support - the emergency employees, while activating self-care strategies could be more valuable in non-emergency employees. Such personalized approaches are already being implanted in programs developed for patients (Bačová, Žiaková, 2017). The results should be further explored and experimentally verified.

\section{References}

Bačová, M., \& Žiaková, E. (2017). Špecializované sociálne poradenstvo ako konkrétna pomoc pre onkologicky chorých pacientov. Acta chemotherapeutica, 26(1-2), 2017.

Bara, A. C., \& Arber, S. (2009). Working shifts and mental health-findings from the British Household Panel Survey (1995-2005). Scandinavian journal of work, environment \& health, 361-367.

Baumann, A., Muijen, M., Gaebel, W. (2010). Mental health and well-being at the workplace-protection and inclusion in challenging times. Copenhagen: The Regional Office for Europe of the World Health Organization.

Madsen, I.E., Nyberg, S.T., Hanson, L.M., et al. (2017). Job strain as a risk factor for clinical depression: systematic review and meta-analysis with additional individual participant data. Psychological Medicine, 8, 1342-1356.

Wu, H., D'Alessio, A. C., Ito, S., Wang, Z., Cui, K., Zhao, K. et al. (2011). Genome-wide analysis of 5-hydroxymethylcytosine distribution reveals its dual function in transcriptional regulation in mouse embryonic stem cells. Genes \& development, 25(7), 679-684. 